\title{
Graphene-mediated exchange coupling between a molecular spin and magnetic substrates
}

\author{
S. Marocchi, ${ }^{1,2, *}$ P. Ferriani, ${ }^{3}$ N. M. Caffrey, ${ }^{3}$ F. Manghi,,${ }^{1,2}$ S. Heinze, ${ }^{3}$ and V. Bellini2 ${ }^{2,4, \dagger}$ \\ ${ }^{1}$ Dipartimento di Fisica, Universitá di Modena e Reggio Emilia, Via Campi 213/A, 41125 Modena, Italy \\ ${ }^{2}$ S3-Istituto di Nanoscienze-CNR, Via Campi 213/A, 41125 Modena, Italy \\ ${ }^{3}$ Institüt für Theoretische Physik und Astrophysik, Christian-Albrecht-Universität zu Kiel, Leibnizstrasse 15, 24098 Kiel, Germany \\ ${ }^{4}$ Istituto di Struttura della Materia (ISM)-Consiglio Nazionale delle Ricerche (CNR), I-34149 Trieste, Italy
}

(Received 6 April 2013; published 8 October 2013)

\begin{abstract}
Using first-principles calculations we demonstrate sizable exchange coupling between a magnetic molecule and a magnetic substrate via a graphene layer. As a model system we consider cobaltocene $\left(\mathrm{CoCp}_{2}\right)$ adsorbed on graphene deposited on $\mathrm{Ni}(111)$. We find that the magnetic coupling is antiferromagnetic and is influenced by the molecule structure, the adsorption geometry, and the stacking of graphene on the substrate. We show how the coupling can be tuned by the intercalation of a magnetic monolayer, such as Fe or Co, between graphene and $\mathrm{Ni}(111)$. We identify the leading mechanism responsible for the coupling to be the spatial and energy matching of the frontier orbitals of $\mathrm{CoCp}_{2}$ and graphene close to the Fermi level. Graphene plays the role of an electronic decoupling layer while allowing effective spin communication between molecule and substrate.
\end{abstract}

DOI: $10.1103 /$ PhysRevB.88.144407

PACS number(s): 71.15.Mb, 75.50.Xx, 68.43.-h, 81.05.ue

\section{INTRODUCTION}

The growing field of organic spintronics capitalizes on the novel functionalities achieved when organic molecules are adsorbed on magnetic substrates. The ability to manipulate and tune these functionalities is an important goal. Several problems remain, however, before such systems can be incorporated into new technological devices. One in particular is the capability to adsorb magnetic molecules on surfaces without any detrimental effects being caused to either its structural or magnetic properties. It is thus vital to choose molecules with maximum structural robustness upon adsorption. ${ }^{1-3}$ To this end, the phthalocyanine (Pc) and porphyrin families are popular choices, due to their planar geometry. ${ }^{4-9}$ However, the strong interaction between the metal ion of such flat molecules and the substrate often modifies its electronic states and can even quench its magnetic moment. ${ }^{10}$ The use of nonplanar molecules, such as metallocenes, can minimize this effect. Metallocenes are composed of a $3 d$ transition-metal ion sandwiched between two cyclopentadienyls (Cps). Depending on the metal ion species, both nonmagnetic and paramagnetic behavior can be found. ${ }^{11}$ The spin of the metal ion is shielded from the surface by the cage formed by the two $\mathrm{Cp}$ rings, reducing the possibility that it will be modified substantially after adsorption.

Another viable route to further decrease an excessive interaction is the intercalation of a decoupling layer between the reactive surface and the molecule. ${ }^{3,12}$ Graphene is an attractive candidate in this regard, primarily due to the unique electronic properties that render it appealing for spintronic applications. It has already been successfully used to decouple Pc molecules from $\operatorname{Ru}(0001)$ and $\operatorname{Ir}(111)$ (Refs. 13 and 14) substrates. It is still an open question, however, if a graphene layer can preserve the magnetic properties of an adsorbed molecule without simultaneously hindering a stable magnetic coupling between the molecular moment and the magnetic substrate. Recent observations of charge transfer at molecule-graphene-Ni(111) interfaces ${ }^{15,16}$ would suggest that a magnetic coupling between a molecule and a substrate through a graphene layer is achievable.
In this work, we predict, by first-principles electronic structure methods, a sizable magnetic coupling for a magnetic molecule adsorbed on a graphene layer deposited on a magnetic substrate, in this case $\mathrm{Ni}(111)$. We consider here cobaltocene $\left(\mathrm{CoCp}_{2}\right)$, for which a theoretical prediction of large charge transfer to graphene has been previously reported. ${ }^{17}$ Furthermore, we propose intercalation of different ferromagnetic metal monolayers, such as $\mathrm{Fe}$ and $\mathrm{Co}$, between graphene and the $\mathrm{Ni}$ substrate as a route to tailor the magnetic coupling. By comparing to the case where cobaltocene is directly adsorbed to $\mathrm{Ni}(111)$, we demonstrate that graphene is crucial to preserving the molecule magnetic moment and acts as an electronic decoupling layer, while allowing effective spin communication between molecule and substrate. Due to the unique electronic properties of graphene, ${ }^{18-20}$ metal-organic systems of this kind could serve as a basis for future spintronic devices.

\section{RESULTS AND DISCUSSION}

Density functional theory (DFT) calculations have been performed using the projector augmented wave method as implemented in the VASP code ${ }^{21,22}$ with the Perdew-BurkeErnzerhof (PBE) exchange correlation functional. ${ }^{23}$ Dispersion interactions have been included according to the DFT-D2 approach. ${ }^{24}$ Further computational details can be found in Appendix A.

Isolated $\mathrm{CoCp}_{2}$ has already been studied extensively by $\mathrm{DFT}^{11}$ and several possible structures have been discussed. We consider here $\mathrm{CoCp}_{2}$ in the energetically lowest highsymmetry configuration $\left(D_{5 h}\right)$ where two possible Jahn-Teller distorted structures characterized by two different electronic states occur (see Appendix B). The probability density of the highest occupied molecular orbital (HOMO) of both of these states, labeled ${ }^{2} B_{2}$ and ${ }^{2} A_{2}$, are plotted in Fig. 1(a). In both cases, the $\mathrm{CoCp}_{2}$ molecule attains a nominal $S=1 / 2$ spin.

The small lattice mismatch $(1.2 \%)$ of graphene and $\mathrm{Ni}(111)$ lattice constant results in pseudomorphic growth and the flat conformation of the graphene layer. ${ }^{25}$ Our DFT calculations show that the bonding between graphene and the $\mathrm{Ni}(111)$ 
(a)

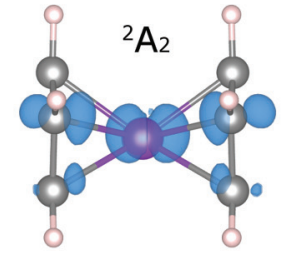

(b)

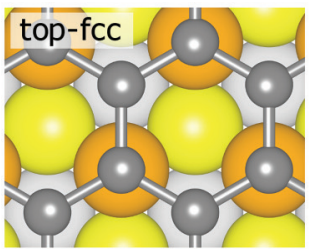

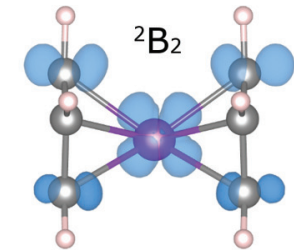

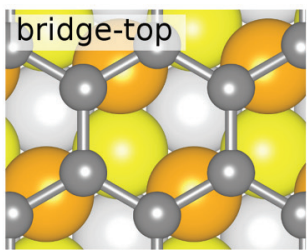

(c)
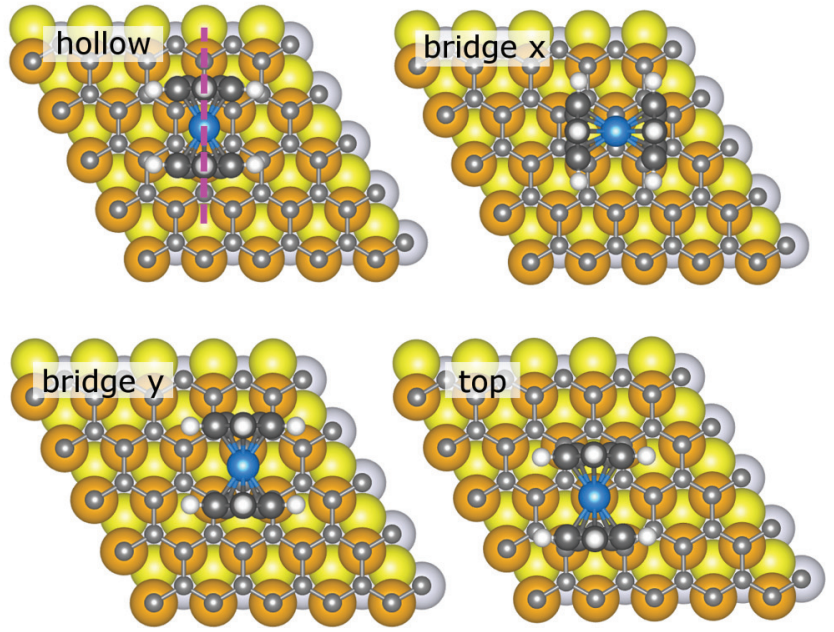

FIG. 1. (Color online) (a) Probability density of the $\mathrm{CoCp}_{2}$ HOMO for the ${ }^{2} A_{2}$ and ${ }^{2} B_{2}$ states. (b) top-fcc and bridge-top stacking of graphene on $\mathrm{Ni}(111)$ (the topmost, second, and third Ni layers are colored orange, yellow, and gray, respectively). (c) Adsorption geometries of the $\mathrm{CoCp}_{2}$ on graphene/Ni(111) for the top-fcc stacking ( $\mathrm{Co}, \mathrm{C}$, and $\mathrm{H}$ atoms in $\mathrm{CoCp}_{2}$ are colored blue, dark gray, and white, respectively).

surface is primarily due to van der Waals (vdW) interactions, with a binding distance of $2.12 \AA$, in good agreement with experiments. $^{26}$

We have taken into account several possible adsorption geometries $\mathrm{CoCp}_{2}$ can assume on graphene/Ni(111), which we labeled as hollow, bridge, and top, depending on the position of the Co atom with respect to the $\mathrm{C}$ atoms below [Fig. 1(c)]. The results are presented in Table I, including the total energy difference $\Delta E$ (with respect to the ground state), the Co (in the $\mathrm{CoCp}_{2}$ molecule)-Ni distance $d$, and the exchange coupling energies $E_{\mathrm{ex}}$, defined as $E_{\mathrm{ex}}=E_{A P}-E_{P}$, where $E_{A P}\left(E_{P}\right)$ is the total energy of the system when the spin moment of the Co atom is antiparallel (parallel) to the one of the Ni slab. Here a negative value of the exchange energy indicates that the cobaltocene's spin moment preferentially orients antiparallel to the Ni magnetization. The lowest energy configuration (first row in Table I, configuration 1) is found when the molecule is adsorbed on the hollow site of graphene, which has a top-fcc stacking on the underlying $\mathrm{Ni}(111)$ substrate. The calculated adsorption energy of this configuration is $\sim 0.64 \mathrm{eV}$,

TABLE I. Total energy difference $\Delta E(\mathrm{meV})$, Co-graphene distance $d(\AA)$, and exchange energy $E_{\text {ex }}(\mathrm{meV})$ for different structural and electronic configurations of $\mathrm{CoCp}_{2}$ on graphene/Ni(111); $\Delta E$ are given in the case of antiparallel alignment of $\mathrm{Co}$ and Ni magnetic moments.

\begin{tabular}{lcrrc}
\hline \hline & Configuration & $\Delta E$ & $d$ & $E_{\mathrm{ex}}$ \\
\hline${ }^{2} \mathrm{~B}_{2}$, top-fcc, hollow & 1 & 0.0 & 6.43 & -9.7 \\
${ }^{2} \mathrm{~A}_{2}$, top-fcc, hollow & 2 & +4.6 & 6.43 & -1.3 \\
${ }^{2} \mathrm{~B}_{2}$, top-fcc, bridge $\mathrm{x}$ & 3 & +55.2 & 6.42 & -4.6 \\
${ }^{2} \mathrm{~B}_{2}$, top-fcc, bridge y & 4 & +74.8 & 6.43 & -8.1 \\
${ }^{2} \mathrm{~B}_{2}$, bridge-top, hollow & 5 & +105.2 & 6.41 & -9.2 \\
${ }^{2} \mathrm{~B}_{2}$, top-fcc, top & 6 & +147.9 & 6.52 & -6.8 \\
\hline \hline
\end{tabular}

somewhere between those indicating physisorption and those indicating chemisorption (see Appendix B). A comparison of the total energies in Table I shows that, except for the case of configuration 2 , all other configurations are strongly energetically unfavorable.

The magnetic ground state shows the molecular spin preferentially aligning antiparallel to the Ni magnetization, with $E_{\text {ex }}$ of the order of $-10 \mathrm{meV}$. This energy is remarkably large if we consider that the distance between the Co and $\mathrm{Ni}$ atoms is approximately $6.4 \AA$. As a comparison, an exchange energy of only $50 \mathrm{meV}$ was found for chemisorbed Fe porphyrin on $\mathrm{Co}(100)$ (Ref. 4), despite the much smaller Fe-Co distance of $\sim 3.5 \AA$. The values of $E_{\mathrm{ex}}$ that we find are high enough to ensure the stability of the spin moments against temperature-induced fluctuations up to more than $100 \mathrm{~K}$, i.e., well above the temperature of the order of a few Kelvins that is employed in state-of-the-art $\mathrm{x}$-ray magnetic dichroism and spin-polarized scanning tunneling microscopy experiments. $^{7,27}$

To elucidate the physical origin of the molecule-substrate exchange coupling we modify independently three possible contributions: the $\mathrm{CoCp}_{2}$ electronic state, the graphene stacking, and the $\mathrm{CoCp}_{2}$ adsorption site. For the first we found that switching from the ${ }^{2} B_{2}$ to the ${ }^{2} A_{2}$ electronic configurations (configurations 1 and 2 in Table I) lowers the exchange energy from -9.7 to $-1.3 \mathrm{meV}$. This considerable decrease can be attributed to the reduced extent of the $\mathrm{CoCp}_{2}$ spin-polarized HOMO [see Fig. 1(a)], which is critical to determining the size of the coupling. Varying the graphene stacking from top-fcc to bridge-top (configurations 1 and 5) does not influence the magnetic coupling in any appreciable way. This is somewhat surprising since the magnetic moment induced on graphene is approximately one order of magnitude smaller in the bridgetop than in the top-fcc stacking, with values of $+0.002 \mu_{B}$ and $-0.03 /+0.02 \mu_{B}$, respectively. We can conclude therefore that the magnetic coupling does not depend on the size of the magnetic moment induced on the graphene atoms. Finally, varying the adsorption site (configurations $1,3,4$, and 6) can change $E_{\text {ex }}$ by up to a factor of two. However, the coupling remains antiferromagnetic in all cases. The relaxed adsorption distance between the $\mathrm{Co}$ and $\mathrm{Ni}$ ions is similar for all the configurations of Table I, i.e., within $0.10 \AA$, and, therefore, cannot play a strong role in the differing exchange energies.

In Fig. 2 we present the spin-polarized local density of states (LDOS) of the system in its ground state (configuration 


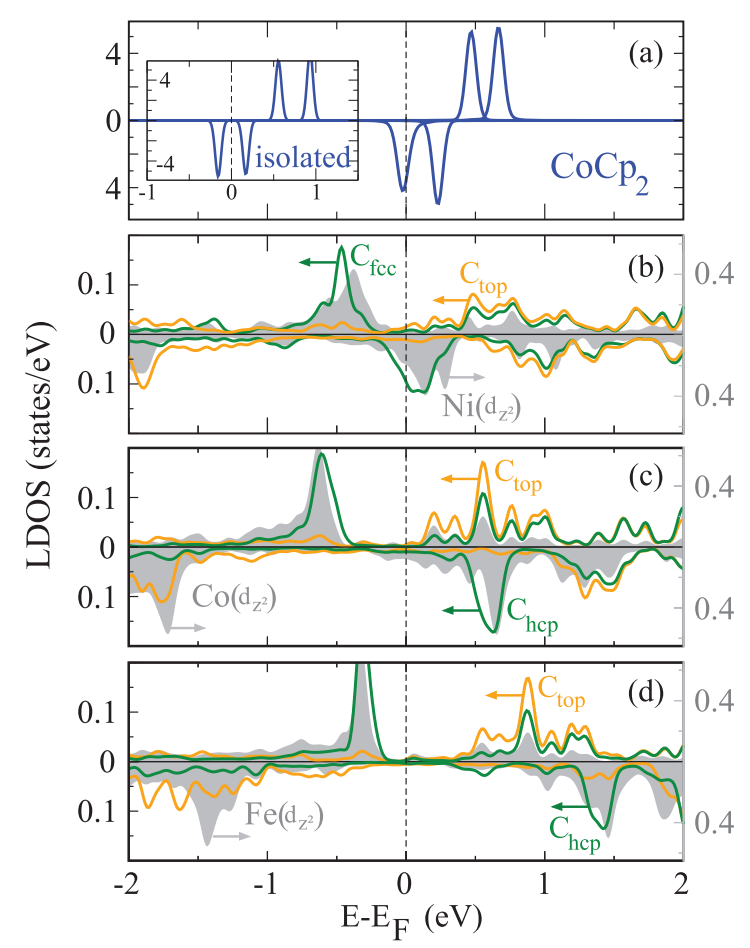

FIG. 2. (Color online) Spin-polarized LDOS of $\mathrm{CoCp}_{2}$ on graphene $/ M / \mathrm{Ni}(111)$ in the antiparallel configuration, with $M=$ $\mathrm{Ni}, \mathrm{Co}$ and Fe: (a) $3 d$ states of Co of $\mathrm{CoCp}_{2}$; (b)-(d) $3 d_{z^{2}}$ states of the $M$ layer atoms and $2 p_{z}$ states of $\mathrm{C}_{\mathrm{top}}$ and $\mathrm{C}_{\mathrm{fcc}}$ (for $M=\mathrm{Ni}$ ) or $\mathrm{C}_{\text {top }}$ and $\mathrm{C}_{\mathrm{hcp}}$ (for $M=\mathrm{Co}, \mathrm{Fe}$; see text). The $d$ states of the $M$ layer are plotted in gray, while graphene $\mathrm{C} p$ states are in orange and dark green. Each curve in panels (b)-(d) is the average over the three atoms of that species closest to the $\mathrm{CoCp}_{2}$ center. Inset in panel (a): $3 d$ states of $\mathrm{Co}$ of $\mathrm{CoCp}_{2}$ for the isolated molecule.

1). Majority (upper panels) and minority (lower panels) states are defined according to the magnetization of the $\mathrm{Ni}(111)$ substrate. Upon adsorption on the surface, we observe a small shift to higher energies of the molecular Co $d$ orbitals with the result that the HOMO is pinned to the Fermi level $\left(\mathrm{E}_{F}\right)$ of the substrate. It also becomes partially depopulated. This is accompanied by a charge transfer of $0.28 e^{-}$from the molecule to the surface and a decrease of the magnetic moment associated to the Co atom from $+0.74 \mu_{B}$ to $+0.47 \mu_{B}$. A hybridization between the $2 p_{z}$ orbital of the graphene atoms and the $3 d_{z^{2}}$ orbital of the $\mathrm{Ni}$ atom is also evident, resulting in the polarization of graphene. Notably, only the $\mathrm{C}_{\mathrm{fcc}}$ atoms exhibit this strong hybridization with the $\mathrm{Ni}$ atoms close to $E_{F}$. The energy overlap between the minority states of graphene and the minority $d$ states of $\mathrm{CoCp}_{2}$ just below $E_{F}$ is responsible for the stabilization of the antiparallel alignment. This energy matching is absent for the parallel alignment, due to the inverted HOMO spin polarization. We can thus conclude that the spin polarization of graphene close to $E_{F}$ determines the sign of the magnetic coupling. This is further corroborated by the analogous situation occurring for configuration 5 , for which both the graphene LDOS around $E_{F}$ and the magnetic coupling are similar to the ones of configuration 1 [see Figs. 3(a) and 3(b)].

Such a dependence suggests that if one can modify the induced spin polarization of graphene in this energy window,
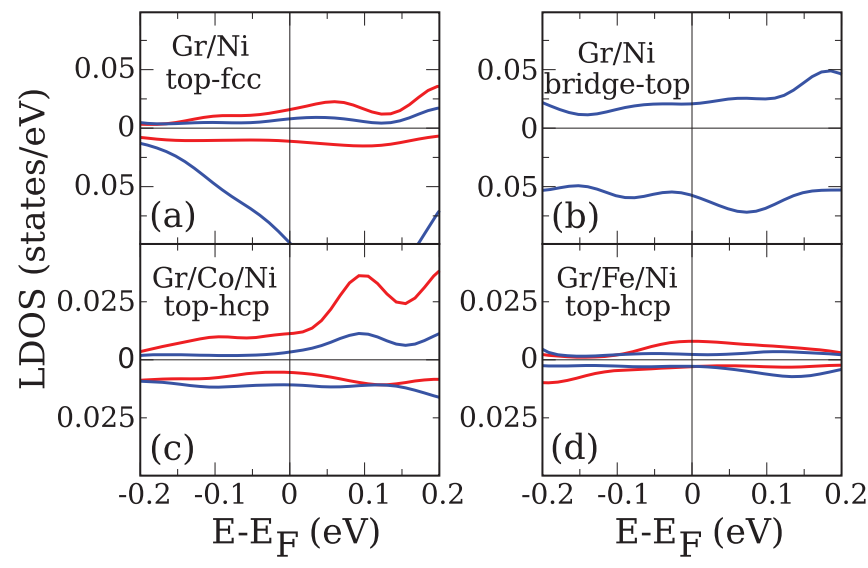

FIG. 3. (Color online) Spin-polarized LDOS at the $\mathrm{C}$ graphene atoms, close to the Fermi level $\left(E_{F}\right)$. (a) $2 p_{z}$ states of $\mathrm{C}_{\text {top }}$ and $\mathrm{C}_{\mathrm{fcc}}$ atoms for configuration 1 of Table I. (b) $2 p_{z}$ states of the only inequivalent $\mathrm{C}$ atom for configuration 5 of Table I. $2 p_{z}$ states of $\mathrm{C}_{\text {top }}$ and $\mathrm{C}_{\mathrm{hcp}}$ atoms for (c) graphene/Co/Ni(111) and (d) graphene/Fe/Ni(111). Each curve is the average over the three atoms of that species closest to the $\mathrm{CoCp}_{2}$ center. The $\mathrm{C}_{\mathrm{top}}$ and $\mathrm{C}_{\mathrm{fcc}} / \mathrm{C}_{\mathrm{hcp}}$ curves are colored red and blue, respectively.

one can modify the magnetic coupling. As a possible realization, we have explored the effect of intercalating different magnetic monolayers ( $\mathrm{Fe}$ and $\mathrm{Co}$ ) between graphene and the $\mathrm{Ni}(111)$ substrate. Experimentally, the intercalation of $\mathrm{Ni}$ and Co monolayers between graphene and $\operatorname{Ir}(111)$ (Refs. 28 and 29) and of Fe between graphene and Ni(111) (Ref. 30) has been successfully achieved. As there are no experimental data for graphene/Co/Ni(111), we have used the same structure as for graphene/Fe/ $\mathrm{Ni}(111)$, which allows for a direct comparison and therefore a clearer insight into the mechanism of the exchange interaction. As discussed in Ref. 30, the intercalated Fe atoms are preferentially placed in the fcc hollow sites of the $\mathrm{Ni}$, following the $\mathrm{Ni}(111)$ stacking. On this substrate, graphene adsorbs in a top-hcp structure, where the two inequivalent graphene $\mathrm{C}$ atoms are placed alternatively above the $\mathrm{Fe}$ atoms and the hcp sites, corresponding to the topmost Ni layer (see Appendix C).

We present in Figs. 2(c) and 2(d) the spin-polarized LDOS in the case of $\mathrm{Fe}$ and $\mathrm{Co}$ intercalation. The values of the corresponding magnetic moments and the exchange energies are listed in Table II. The magnitude of the spin moment in the interface metal $(M)$ layer increases as one goes from $\mathrm{Ni}$ to $\mathrm{Co}$ to $\mathrm{Fe}$ and, due to hybridization, this increase also occurs for the moments induced on the $\mathrm{C}$ atoms, i.e., $m_{C}^{\text {top }}$ and $m_{C}^{\mathrm{fcc}}$.

TABLE II. Magnetic moments of the two nonequivalent atoms of graphene $m_{C}^{\text {top }}\left(\mu_{B}\right)$ and $m_{C}^{\mathrm{fcc} / \mathrm{hcp}}\left(\mu_{B}\right)$, the interface metal monolayer $m_{M}\left(\mu_{B}\right)$, and the exchange energies $E_{\mathrm{ex}}(\mathrm{meV})$ for $\mathrm{CoCp}_{2}$ on graphene $/ M / \mathrm{Ni}(111)(M=\mathrm{Ni}, \mathrm{Co}, \mathrm{Fe})$.

\begin{tabular}{llll}
\hline \hline & $\mathrm{gr} / \mathrm{Ni} / \mathrm{Ni}$ & $\mathrm{gr} / \mathrm{Co} / \mathrm{Ni}$ & $\mathrm{gr} / \mathrm{Fe} / \mathrm{Ni}$ \\
\hline$m_{C}^{\text {top }}$ & -0.02 & -0.04 & -0.05 \\
$m_{C}^{\mathrm{fc} / \mathrm{hcp}}$ & +0.03 & +0.04 & +0.04 \\
$m_{M}$ & +0.47 & +1.52 & +2.39 \\
$E_{\mathrm{ex}}$ & -9.7 & -2.3 & +2.0 \\
\hline \hline
\end{tabular}


Surprisingly, the magnetic coupling is not found to increase in line with the magnetic moment and in fact decreases. We can identify a trend for the exchange energy between $\mathrm{CoCp}_{2}$ and the investigated substrate from large antiferromagnetic $\left(E_{\mathrm{ex}}=\right.$ $-9.7 \mathrm{meV})$ for graphene/Ni(111), to weak antiferromagnetic $\left(E_{\mathrm{ex}}=-2.3 \mathrm{meV}\right)$ for graphene/Co/Ni(111) and weak ferromagnetic $\left(E_{\mathrm{ex}}=+2.0 \mathrm{meV}\right)$ for graphene/Fe/Ni(111). The energy matching between the HOMO of $\mathrm{CoCp}_{2}$ and the $p_{z}$ states of the carbon atoms, which drives the coupling between the molecule and substrate, is disrupted by the intercalation of the metal layer. The minority $d_{z^{2}}$ states of the Co layer lie at higher energies than those of $\mathrm{Ni}$ and the Fe states are found at even higher energies. Due to hybridization, the $p_{z}$ orbitals of the graphene atoms are similarly shifted to higher energies. This reduces (for Co intercalation) and finally prevents (for Fe intercalation) the energy matching of the $\mathrm{C}$ states with the spin-polarized HOMO of $\mathrm{CoCp}_{2}$ with a resultant decrease in the magnetic coupling.

Figures 3(c) and 3(d) confirm this trend. For the graphene/Co/Ni(111) system, indeed the LDOS of the $\mathrm{C}$ atoms integrated in the region within $0.1 \mathrm{eV}$ below $E_{F}$ in the minority spin channel is somewhat larger than the one in the majority channel and so the matching with the $\mathrm{CoCp}_{2} \mathrm{HOMO}$

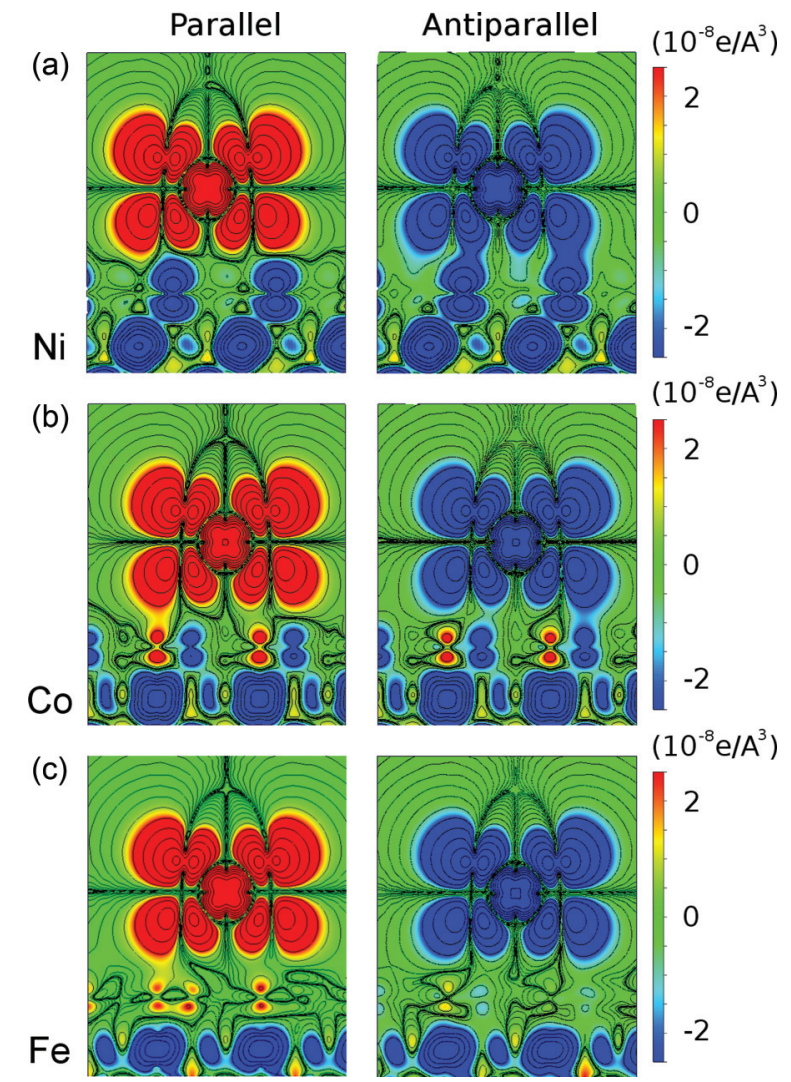

FIG. 4. (Color online) Cross-sectional plots of the local magnetization density integrated from $-0.1 \mathrm{eV}$ to the Fermi level of $\mathrm{CoCp}_{2}$ on (a) graphene/Ni(111) in top-fcc stacking, (b) graphene/Co/Ni(111), and (c) graphene/Fe/Ni(111). Left (right) panels refer to the parallel (antiparallel) configuration. The cross-sectional plane, indicated as purple broken line in Fig. 1(c) (hollow adsorption site), cuts through the Co atom and is perpendicular to both the substrate and the $\mathrm{Cp}$ rings. results in a small antiparallel coupling. On the other hand, for graphene/Fe/ $\mathrm{Ni}(111)$ there is a slight excess of majority spin in the same energy range, which is consistent with the weak parallel coupling between the molecule and the substrate spins.

Cross sectional plots of the magnetization density, i.e., the difference between the spin up and down charge densities, close to the Fermi level are given in Fig. 4. In panel (a) the spatial matching of the $\mathrm{CoCp}_{2} \mathrm{HOMO}$ with the $p_{z}$ orbitals of the $\mathrm{C}_{\mathrm{fcc}}$ atom of graphene adsorbed on $\mathrm{Ni}(111)$ is evident in the antiparallel alignment where spin density lobes from the molecule and the surface atoms merge. In contrast, it is absent in the parallel alignment resulting in a negative exchange energy. For $\mathrm{CoCp}_{2}$ on graphene/Co/Ni [Fig. 4(b)] there is an excess of majority spin for the $\mathrm{C}_{\text {top }}$ atoms and of minority spin for the $\mathrm{C}_{\mathrm{hcp}}$ atoms which almost cancel each other. However, a small preference towards communication through the minority spin is suggested by the plot in accordance with the weak antiferromagnetic coupling. For the Fe intercalated layer [Fig. 4(c)], the spin density in the graphene indicates spin communication for the parallel alignment, but not for the antiparallel alignment, explaining the positive exchange energy. The analysis performed for configuration 5 of Table I (see Fig. 5) reveals a scenario similar to the one of Fig. 4(a) and is consistent with an exchange coupling of similar size.

To unambiguously determine the role of graphene in mediating the interaction between $\mathrm{CoCp}_{2}$ and the magnetic substrate, we considered the situation when $\mathrm{CoCp}_{2}$ is adsorbed directly on the Ni(111) surface (see Appendix B). In this case, the molecule is chemisorbed with a distance between the Co and $\mathrm{Ni}$ atoms of $d=4.30 \AA$ and an adsorption energy of $\sim 1.3 \mathrm{eV}$, i.e., about twice as large the one found in the presence of the graphene layer. Also the charge transfer from $\mathrm{CoCp}_{2}$ to $\mathrm{Ni}$ approximately doubles from 0.28 to 0.64 electrons if the spacing layer is removed. This indicates that graphene plays a crucial role in the electronic decoupling of the molecule and the substrate, and can facilitate the preservation of the structural integrity of such molecules upon deposition on metallic surfaces. As a matter of fact, it has been previously shown that deposition of metallocenes on metallic surfaces is a difficult process ${ }^{31}$ and can, in some cases, result in the complete dissociation of the molecule. ${ }^{32,33}$ Interestingly, the magnetic moment of cobaltocene is fully quenched when it is directly adsorbed on Ni(111). This clearly demonstrates that graphene is essential to preserve the magnetic properties of the molecule.

$\mathrm{Ni}$
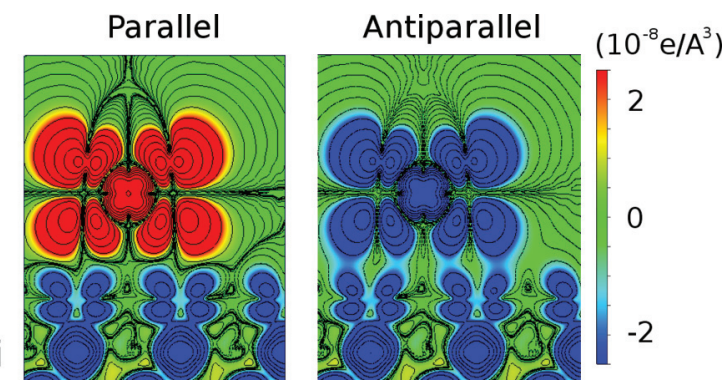

FIG. 5. (Color online) Cross-sectional plots of the local magnetization density integrated from $-0.1 \mathrm{eV}$ to the Fermi level of $\mathrm{CoCp}_{2}$ on graphene/ $\mathrm{Ni}(111)$ in bridge-top stacking. Left (right) panels refer to the parallel (antiparallel) configuration. 


\section{SUMMARY AND CONCLUSION}

In conclusion, our work demonstrates that graphene plays a vital role in determining the interaction between a magnetic molecule and a ferromagnetic substrate. As a test case system in which to study these effects, we consider $\mathrm{CoCp}_{2}$ adsorbed on a graphene layer grown on a magnetic Ni(111) substrate. We find that graphene behaves as an effective electronic decoupling layer yet allowing spin communication between the molecule and the substrate. The magnetic coupling is antiferromagnetic and the strength of the coupling can be manipulated by changing the structural details of the adsorption. We also show that it is possible to tune the sign of the coupling by intercalating a magnetic monolayer between graphene and the Ni substrate.

Note added: Recently, Ref. 34 was published, where a study of the magnetic coupling between Co-octaethylporphyrin molecules and graphene/Ni(111) is presented.

\section{ACKNOWLEDGMENTS}

We thank Dr. Carlo Pignedoli for fruitful discussions. This work has been supported by FCRM "THE-SIMS," the Deutsche Forschungsgemeinschaft via the SFB 677, the European Science Foundation (ESF) under the EUROCORES Program Euro-GRAPHENE, and the German Academic Exchange Service (DAAD). We acknowledge the CINECA and HLRN centers for granting the high-performance computing resources.

\section{APPENDIX A: COMPUTATIONAL DETAILS}

We performed preliminary calculations on a $p(1 \times 1)$ hexagonal supercell to obtain geometry optimized graphene/ $M / \mathrm{Ni}(111)(M=\mathrm{Ni}, \mathrm{Co}, \mathrm{Fe})$ structures and a density-of-states analysis of the systems. In this first part of our work we used a system containing one layer (two C atoms) of graphene and a four-layer metal slab. An optimized $\Gamma$-centered $k$-point grid of $17 \times 17 \times 1$ has been used for these small supercell calculations. The graphene layer and the top layer of metal were relaxed along the $z$ axis. The three bottom layers of $\mathrm{Ni}$ were fixed at the bulk geometry with a lattice parameter of $2.49 \AA$ that corresponds to the PBE bulk optimized $\mathrm{Ni}-\mathrm{Ni}$ distance.

In order to verify the accuracy of this geometry we compared to a calculation in which the system has been modeled using the $p(1 \times 1)$ unit cell with a six-layer metal slab and allowing the graphene layer and the three topmost layers of Ni to relax in all directions, i.e., four substrate layers have been fully relaxed. We found that the $\mathrm{C}$-Ni distance changes as little as $0.001 \AA$ as compared to the calculations reported in our article. The distance between the Ni surface and subsurface layers varies by $0.004 \AA$. The modification to the magnetic moments is only $0.002 \mu_{B}$ for the $\mathrm{C}$ atoms and $0.019 \mu_{B}$ for the surface $\mathrm{Ni}$ atom, consistent with the tiny geometry variations. Such changes are minor and they do not affect the interaction between molecule and substrate, allowing us to use a four-layer rather than a six-layer Ni slab. The geometries of these small cells have been replicated in plane in order to build the bigger $p(5 \times 5)$ supercell containing not only the graphene-metal slab but also the $\mathrm{CoCp}_{2}$ molecule. In this second set of calculations, the graphene and metal layers were fixed and only the $\mathrm{CoCp}_{2}$ was fully relaxed. A $\Gamma$-centered grid of $3 \times 3 \times 1 k$ points has been used. Minimization proceeded until forces were lower than $0.01 \mathrm{eV} \AA^{-1}$.

We included in our calculations the vdW dispersion interactions. It is known that the nonlocal exchange-correlation energy functional (vdW-DF) results in an equilibrium distance between graphene and the topmost Ni layer larger than $3.5 \AA$ (Ref. 35) for all the calculated structures, in disagreement with experiments. Thus, we employed the semiempirical potential DFT-D2 of Grimme. ${ }^{24}$ With this method the estimate of the interaction energy is less reliable as compared to the vdW-DF; however, the typical errors never exceed $20 \%$ (Ref. 24). On the other hand, the DFT-D2 gives trustworthy equilibrium distances. On average, the distances obtained with this method for small aromatic systems underestimate by about $5 \%$ the experimental values. ${ }^{24}$ In light of the fact that in our calculations it is essential to get the equilibrium distances as reliable as possible, we decided to use the semiempirical potential DFT-D2. Charge transfers were calculated using the Bader analysis. ${ }^{36}$

We checked the influence of an on-site Coulomb interaction on the exchange coupling energies $E_{\mathrm{ex}}$ for the cases of graphene/ $\mathrm{Ni}(111)$ and graphene/Fe/Ni(111). Imposing static correlation effects on the $d$ electrons of $\mathrm{Co}$ in $\mathrm{CoCp}_{2}$ (values of $U-J=2$ and $4 \mathrm{eV}$ have been tested), the magnetic coupling for $\mathrm{CoCp}_{2}$ on graphene/Ni(111) changes from -9.7 to $-10.5 \mathrm{meV}$ while for the case of graphene/Fe/Ni(111) substrate it is not modified to any extent. We conclude that the employed GGA exchange correlation potential describes the magnetic properties of the investigated systems with sufficient accuracy.

\section{APPENDIX B: COBALTOCENE ELECTRONIC STRUCTURE AND ADSORPTION ON Ni(111) AND GRAPHENE/Ni(111)}

We plot in Fig. 6 the two possible structures of a metallocene molecule, $D_{5 h}$ and $D_{5 d}$, named after their point group symmetry. In the former the two pentagons of the $\mathrm{Cp}$ rings are symmetric, while in the latter they are rotated by $180^{\circ}$. Total energy calculations show that $D_{5 h}$ symmetry is energetically favored for isolated $\mathrm{CoCp}_{2}$ molecules, with respect to $D_{5 d}$. The

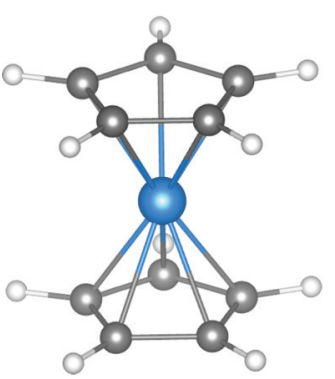

(a)

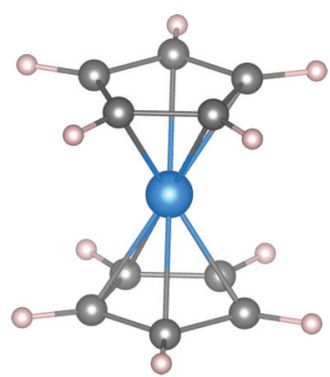

(b)
FIG. 6. (Color online) (a) $\mathrm{CoCp}_{2}$ in the symmetry configuration $D_{5 h}$. (b) $\mathrm{CoCp}_{2}$ in the symmetry configuration $D_{5 d}$. Co, C, and $\mathrm{H}$ atoms in $\mathrm{CoCp}_{2}$ are colored blue, gray, and white, respectively. 
crystal field produced by the $\mathrm{Cp}$ rings split the $3 d$ orbitals of Co. If we consider the axis of the molecule as our $z$ axis, the seven $3 d$ electrons are split in (i) two electrons in a singlet orbital derived from $d_{z^{2}}$, (ii) four electrons in a doubly degenerate orbitals derived from $d_{x y}$ and $d_{x^{2}-y^{2}}$, (iii) one electron in a doubly degenerate orbitals derived from $d_{y z}$ and $d_{x z}$. The $D_{5 h}$ symmetry is distorted to remove the degeneracy of the frontier orbitals, according to the Jahn-Teller effect, so the symmetry is reduced to $\mathrm{C}_{2 v}$. There are two possible distortions and thus two possible electronic states; depending on whether the $\mathrm{Cp}$ rings tilt slightly toward the molecular center or outwards, the ${ }^{2} B_{2}$ or the ${ }^{2} A_{2}$ electronic states are respectively produced, as shown in Fig. 1(a). In the ${ }^{2} B_{2}$ state, the HOMO has $d_{y z}$ character while in the ${ }^{2} A_{2}$ state it has $d_{x z}$ character.

The adsorption of $\mathrm{CoCp}_{2}$ on graphene/Ni(111) can be regarded as strong physisorption as inferred from the fact that (i) the smallest distance between the $\mathrm{H}$ atoms of $\mathrm{CoCp}_{2}$ and the $\mathrm{C}$ atoms of graphene is $\sim 2.4 \AA$, (ii) there is no appreciable distortion of the structure of $\mathrm{CoCp}_{2}$ upon deposition on the surface, and (iii) the $\mathrm{C}-\mathrm{C}, \mathrm{C}-\mathrm{Ni}$, and $\mathrm{C}-\mathrm{H}$ bonds are nonpolar (or only weakly polar) excluding the formation of hydrogen bonds. The configuration where the $\mathrm{CoCp}_{2}$ is oriented with its axis perpendicular to the surface and in the top adsorption site, i.e., where the Co atom is directly above one of the graphene $\mathrm{C}_{\text {top }}$ atom, is less stable than the configuration with the $\mathrm{CoCp}_{2}$ axis parallel to the substrate surface (configuration 1 in Table I) by $40 \mathrm{meV}$. The minimum distance is $3.0 \AA$, as compared to $2.4 \AA$.

When the $\mathrm{CoCp}_{2}$ is adsorbed directly on the $\mathrm{Ni}(111)$ surface the magnetic moment of $\mathrm{CoCp}_{2}$ is fully quenched. This can be recovered by rigidly shifting the molecule away from the surface by $1 \AA$, whereby the Co ion attains a magnetic moment of $+0.26 \mu_{B}$. As for the case including the graphene layer, the magnetic coupling is antiparallel, albeit weakly $\left(E_{\mathrm{ex}}=\right.$ $-0.4 \mathrm{meV})$. A further rigid shift of the molecule by $1 \AA$ results
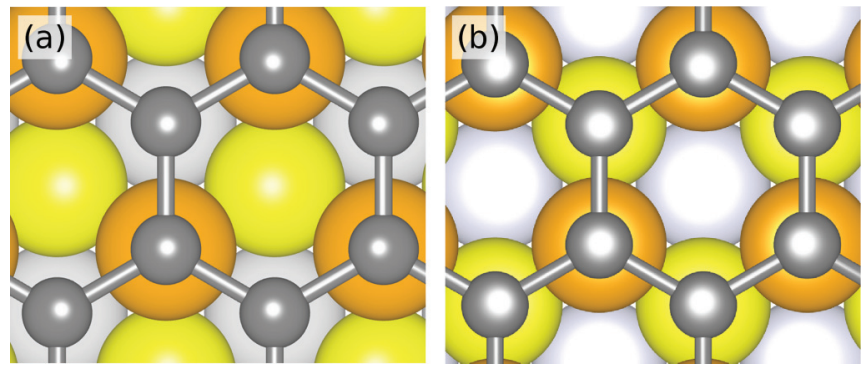

FIG. 7. (Color online) (a) top-fcc stacking of graphene on $\mathrm{Ni}(111)$. (b) top-hcp stacking of graphene on $M / \mathrm{Ni}(111)(M=$ $\mathrm{Fe}, \mathrm{Co})$. The topmost, second, and third metal layers are colored orange, yellow, and gray, respectively.

in an increase of the Co magnetic moment to $+0.35 \mu_{B}$, while the exchange coupling becomes negligibly small.

\section{APPENDIX C: GRAPHENE STACKING FOR FE AND CO INTERCALATION}

The surface lattice constant of the $\mathrm{Ni}(111)$ is $2.49 \AA$, with a very small lattice mismatch, i.e., $1.2 \%$, with the one of graphene $(2.46 \AA)$. In the case of the intercalated Fe or Co monolayer, the lattice mismatch increases, yet a single layer is stable and does not lead to relevant modification in the graphene structure. The energetically favored top-fcc structure for graphene/Ni(111) (a) is compared with the top-hcp structure (b) attained for graphene/ $M / \mathrm{Ni}(111)$, with $M=\mathrm{Fe}$ and $\mathrm{Co}$; first (topmost, orange), second (yellow), and third (light gray) metallic layer atoms below graphene atoms (dark gray) are depicted in Fig. 7. This figure was obtained using VESTA (Ref. 37). We found for graphene/Fe/Ni(111) and graphene/Co/Ni(111) a distance between graphene and the topmost metal layer of $\sim 2.09$ and $\sim 2.08 \AA$, respectively, close to the one of graphene/Ni(111).

\footnotetext{
*simone.marocchi@unimore.it

${ }^{\dagger}$ valerio.bellini@unimore.it

${ }^{1}$ M. Mannini, F. Pineider, P. Sainctavit, C. Danieli, E. Otero,

C. Sciancalepore, A. M. Talarico, M.-A. Arrio, A. Cornia,

D. Gatteschi et al., Nat. Mater. 8, 194 (2009).

${ }^{2}$ A. Ghirri, V. Corradini, V. Bellini, R. Biagi, U. del Pennino, V. De Renzi, J. C. Cezar, C. A. Muryn, G. A. Timco, R. E. P. Winpenny et al., ACS Nano 5, 7090 (2011).

${ }^{3}$ S. Kahle, Z. Deng, N. Malinowski, C. Tonnoir, A. Forment-Aliaga, N. Thontasen, G. Rinke, D. Le, V. Turkowski, T. S. Rahman et al., Nano Lett. 12, 518 (2012).

${ }^{4}$ H. Wende, M. Bernien, J. Luo, C. Sorg, N. Ponpandian, J. Kurde, J. Miguel, M. Piantek, X. Xu, P. Eckhold et al., Nat. Mater. 6, 516 (2007).

${ }^{5}$ S. Javaid, M. Bowen, S. Boukari, L. Joly, J.-B. Beaufrand, X. Chen, Y. J. Dappe, F. Scheurer, J.-P. Kappler, J. Arabski et al., Phys. Rev. Lett. 105, 077201 (2010).

${ }^{6}$ C. Wäckerlin, D. Chylarecka, A. Kleibert, K. Müller, C. Iacovita, F. Nolting, T. A. Jung, and N. Ballav, Nat. Commun. 1, 61 (2010).
}

${ }^{7}$ A. Lodi Rizzini, C. Krull, T. Balashov, J. J. Kavich, A. Mugarza, P. S. Miedema, P. K. Thakur, V. Sessi, S. Klyatskaya, M. Ruben et al., Phys. Rev. Lett. 107, 177205 (2011).

${ }^{8}$ J. Schwöbel, Y. Fu, J. Brede, A. Dilullo, G. Hoffmann, S. Klyatskaya, M. Ruben, and R. Wiesendanger, Nat. Commun. 3, 953 (2012).

${ }^{9}$ E. Annese, F. Casolari, J. Fujii, and G. Rossi, Phys. Rev. B 87, 054420 (2013).

${ }^{10}$ J. Brede, N. Atodiresei, S. Kuck, P. Lazić, V. Caciuc, Y. Morikawa, G. Hoffmann, S. Blügel, and R. Wiesendanger, Phys. Rev. Lett. 105, 047204 (2010).

${ }^{11} \mathrm{Z} . \mathrm{Xu}, \mathrm{Y} . \mathrm{Xie}, \mathrm{W}$. Feng, and H. Schaefer, III, J. Phys. Chem. A 107, 2716 (2003).

${ }^{12}$ J. Repp, G. Meyer, S. M. Stojković, A. Gourdon, and C. Joachim, Phys. Rev. Lett. 94, 026803 (2005).

${ }^{13}$ J. Mao, H. Zhang, Y. Jiang, Y. Pan, M. Gao, W. Xiao, and H.-J. Gao, J. Am. Chem. Soc. 131, 14136 (2009).

${ }^{14}$ M. Scardamaglia, S. Lisi, S. Lizzit, A. Baraldi, R. Larciprete, C. Mariani, and M. G. Betti, J. Phys. Chem. C 117, 3019 (2013). 
${ }^{15}$ W. Dou, S. Huang, R. Q. Zhang, and C. S. Lee, J. Chem. Phys. 134, 094705 (2011).

${ }^{16}$ J. Uihlein, H. Peisert, M. Glaser, M. Polek, H. Adler, F. Petraki, R. Ovsyannikov, M. Bauer, and T. Chassé, J. Chem. Phys. 138, 081101 (2013).

${ }^{17}$ Y. Li, X. Chen, G. Zhou, W. Duan, Y. Kim, M. Kim, and J. Ihm, Phys. Rev. B 83, 195443 (2011).

${ }^{18}$ A. Candini, S. Klyatskaya, M. Ruben, W. Wernsdorfer, and M. Affronte, Nano Lett. 11, 2634 (2011).

${ }^{19}$ S. M. Avdoshenko, I. N. Ioffe, G. Cuniberti, L. Dunsch, and A. A. Popov, ACS Nano 5, 9939 (2011).

${ }^{20}$ J. Maassen, W. Ji, and H. Guo, Nano Lett. 11, 151 (2010).

${ }^{21}$ G. Kresse and J. Furthmüller, Phys. Rev. B 54, 11169 (1996).

${ }^{22}$ G. Kresse and D. Joubert, Phys. Rev. B 59, 1758 (1999).

${ }^{23}$ J. P. Perdew, K. Burke, and M. Ernzerhof, Phys. Rev. Lett. 77, 3865 (1996).

${ }^{24}$ S. Grimme, J. Comput. Chem. 27, 1787 (2006).

${ }^{25}$ L. V. Dzemiantsova, M. Karolak, F. Lofink, A. Kubetzka, B. Sachs, K. von Bergmann, S. Hankemeier, T. O. Wehling, R. Frömter, H. P. Oepen et al., Phys. Rev. B 84, 205431 (2011).

${ }^{26}$ Y. Gamo, A. Nagashima, M. Wakabayashi, and M. Terai, Surf. Sci. 374, 61 (1997).
${ }^{27}$ R. Wiesendanger, Rev. Mod. Phys. 81, 1495 (2009).

${ }^{28}$ D. Pacilé, P. Leicht, M. Papagno, P. M. Sheverdyaeva, P. Moras, C. Carbone, K. Krausert, L. Zielke, M. Fonin, Y. S. Dedkov et al., Phys. Rev. B 87, 035420 (2013).

${ }^{29}$ R. Decker, J. Brede, N. Atodiresei, V. Caciuc, S. Blügel, and R. Wiesendanger, Phys. Rev. B 87, 041403 (2013).

${ }^{30}$ M. Weser, E. N. Voloshina, K. Horn, and Y. S. Dedkov, Phys. Chem. Chem. Phys. 13, 7534 (2011).

${ }^{31}$ B. W. Heinrich, L. Limot, M. V. Rastei, C. Iacovita, J. P. Bucher, D. M. Djimbi, C. Massobrio, and M. Boero, Phys. Rev. Lett. 107, 216801 (2011).

${ }^{32}$ K.-F. Braun, V. Iancu, N. Pertaya, K.-H. Rieder, and S.-W. Hla, Phys. Rev. Lett. 96, 246102 (2006).

${ }^{33}$ J. Choi and P. Dowben, Surf. Sci. 600, 2997 (2006).

${ }^{34}$ C. F. Hermanns, K. Tarafder, M. Bernien, A. Krüger, Y.-M. Chang, P. M. Oppeneer, and W. Kuch, Adv. Mater. 25, 3473 (2013).

${ }^{35}$ M. Vanin, J. J. Mortensen, A. K. Kelkkanen, J. M. Garcia-Lastra, K. S. Thygesen, and K. W. Jacobsen, Phys. Rev. B 81, 081408 (2010).

${ }^{36}$ G. Henkelman, A. Arnaldsson, and H. Jónsson, Comp. Mater. Sci. 36, 354 (2006).

${ }^{37}$ K. Momma and F. Izumi, J. Appl. Cryst. 41, 653 (2008). 